\title{
Selección del hábitat de nidificación de la lechuza vizcachera (Athene cunicularia) en agroecosistemas de la Pampa Ondulada
}

\author{
Pamela E. Pairo ${ }^{1, \varpi ; ~ L u c a s ~ M . ~ L e v e a u ~}{ }^{2} \&$ M. Isabel BellocQ ${ }^{2}$ \\ ${ }^{I}$ Centro de Investigaciones Científicas y Transferencia de Tecnología a la Producción (CICYTTP-CONICET). Diamante, Entre \\ Rios, Argentina. ${ }^{2}$ IEGEBA (CONICET-UBA), Ciudad Autónoma de Buenos Aires, Argentina.
}

\begin{abstract}
Resumen. La lechuza vizcachera es una rapaz típica de la Región Pampeana, que construye su nido en madrigueras en el suelo. Dado el impacto negativo de la expansión del cultivo de soja sobre la diversidad de aves, resulta clave estudiar su efecto sobre la selección y las características del hábitat en múltiples escalas. Se investigó el uso del hábitat de nidificación antes y después del brote de la soja de primera y la selección del hábitat de nidificación después del brote de la soja, en tres escalas espaciales: nido (hábitat donde la lechuza vizcachera construye su nido), sitio de nidificación (círculo de radio $50 \mathrm{~m}$ ) y área de acción (círculo de radio $600 \mathrm{~m}$ ). Se recorrieron caminos secundarios localizando nidos de lechuzas y se establecieron 23 puntos al azar para analizar la selección de hábitat. El uso del hábitat varió según la escala y la etapa del cultivo. Antes del brote de la soja, $63 \%$ de los nidos se ubicaron en el interior de campos ganaderos, mientras que después, 55\% de los nidos se encontraron en los bordes. En la escala de sitio de nidificación, la cobertura de los tipos de hábitat y la distancia al alambrado fueron similares antes y después del brote de la soja. En la escala de área de acción, antes del brote de la soja, la lechuza vizcachera nidificó rodeada de áreas con rastrojo; después, en áreas con cultivo de soja. La lechuza vizcachera seleccionó los bordes de campos de cultivo para ubicar sus nidos, evitó la cercanía de caminos y arboledas en la escala de sitio, y en la escala de área de acción nidificó cerca de alambrados y evitó las arboledas. Recomendamos mantener los bordes de cultivo con vegetación no manejada para proveer hábitat de nidificación para la lechuza vizcachera.
\end{abstract}

[Palabras clave: sistemas agrícolas, soja, nidificación]

\begin{abstract}
Nesting site selection by the Burrowing Owl (Athene cunicularia) in Rolling Pampa agroecosystems. The Burrowing owl is a common raptor species in Pampean agrosystems of Argentina, that builds nests in burrows under the ground. Given the negative effect of expanding soybean crops on avian diversity, it is key to study that effect on habitat selection at multiples scales. We investigated the use of nesting habitat before and after the growing of soybean shoots and nesting site selection after the growing of soybean shoots, at three spatial scales: nest scale (habitat type where the nest was located), nesting site scale (a circle of $50 \mathrm{~m}$ radius centered on the nest) and home range (a circle of $600 \mathrm{~m}$ radius). Surveys were conducted on secondary roads, searching for nests, and 23 random points were established on the same roads to evaluate nest site selection. Habitat use changed with the spatial scale and stage of crop development. Before the soybean shooting, $63 \%$ of nests were located into livestock fields, whereas after the soybean shooting, 55\% of nests were located in field edges. At the nesting site scale, the cover of habitat types and distance to fences were similar before and after soybean shooting. At the home range scale, Burrowing Owl nested in stubble area and in cropfields before and after soybean shooting, respectively. The Burrowing Owl selected field edges to locate the nest, avoided the proximity of roads and woodlots at the nesting site scale, and selected home ranges close to fences and avoiding trees. We recommend maintaining edges of cropfields with unmanaged vegetation to provide nesting habitat for burrowing owls.
\end{abstract}

[Keywords: habitat selection, agrosystem, soybean, nesting site]

\section{INTRODUCCIÓN}

El hábitat que una especie utiliza es una de las dimensiones del nicho más relevantes de su historia de vida, y el conocimiento de la selección por distintos tipos de hábitats es fundamental para evaluar potenciales respuestas a disturbios e implementar programas de conservación. Block and Brennan (1993) definen el "uso de hábitat" como el grupo de características físicas del ambiente usadas por los individuos de una especie para su supervivencia y reproducción. La "selección de hábitat" se refiere a un proceso

Editor asociado: Pedro Blendinger jerárquico de respuestas conductuales que puede resultar en el uso desproporcionado de los hábitats y que influyen en la supervivencia y la aptitud de individuos a distintas escalas (Johnson 1980). En este trabajo utilizamos "selección de hábitat" para indicar el resultado de ese proceso y no el proceso en sí mismo. La selección que realiza un individuo puede ser evaluada comparando el hábitat usado con el hábitat no usado o con el disponible (Jones 2001). Los criterios de selección son diferentes según la escala espacial considerada, que variaría de forma jerárquica desde el área 
de distribución geográfica hasta el área de acción del individuo (Manly et al. 1993). En los últimos años aumentó la cantidad de estudios de selección de hábitat de aves en diferentes escalas espaciales dado que contribuyen a entender la distribución de los organismos (Thiollay and Rahman 2002; Cueto 2006; Piorecky and Prescott 2006; Boscolo and Metzger 2009; Stevens et al. 2011; Baladrón et al. 2016).

La lechuza vizcachera (Athene cunicularia) es una rapaz de áreas abiertas, con amplia distribución en América. Habita en el oeste de Canadá, el oeste de los Estados Unidos, México, América Central y América del Sur (Haug et al. 1993). En América del Norte, sus poblaciones decrecieron de forma significativa (Jones and Bock 2002; Chipman et al. 2008) principalmente a causa de la fragmentación del hábitat, del uso de pesticidas y del control de los mamíferos cavadores (Holroyd et al. 2001; Conway et al. 2006; Lantz et al. 2007). Por este motivo, en Norteamérica se estudió la selección del hábitat de nidificación en diferentes ambientes y escalas (Lantz et al. 2007; Stevens et al. 2011; Crowe et al. 2013). En la Argentina, la mayoría de los estudios se realizaron sobre la dieta de la lechuza vizcachera en agroecosistemas (Bellocq 1988; Cavalli et al. 2014; pero ver Bellocq 1993) y en ambientes áridos (Andrade et al. 2010; Carevic et al. 2013). En cambio, la selección del hábitat fue menos estudiada. En investigaciones llevadas a cabo en la Pampa Inundable se encontró que la lechuza vizcachera evitó sitios con vegetación alta y cultivos a escala de territorio, mientras que en la escala de paisaje seleccionó áreas con bordes y evitó áreas con disturbio antrópico (Baladrón et al. 2016).

Estudios realizados en la Pampa argentina indican que la lechuza vizcachera construye sus propias madrigueras (Hudson 1920; Canevari et al. 1991) y también utiliza las cuevas construidas por las vizcachas (Lagostomus maximus), a lo que debe su nombre común, las cuevas de armadillos (Chaetophractus villosus) y las construcciones humanas (Machicote et al. 2004; Martinelli 2010). Al ser un predador tope cuya dieta incluye pequeños mamíferos (Bellocq 1987; De Tommaso et al. 2009; Cavalli et al. 2014), las lechuzas regulan poblaciones de roedores vectores de enfermedades que afectan al hombre, como el Síndrome Pulmonar de Hantavirus (Palma et al. 2012). Por lo tanto, es fundamental entender qué factores ambientales determinan las dimensiones de su hábitat de nidificación.

Los pastizales naturales de la Pampa Ondulada fueron muy degradados y reemplazados; primero, por la ganadería; luego, por la agricultura (con cultivos de trigo, maíz, sorgo, girasol y soja), y posteriormente, la dominancia de los cultivos de soja en los paisajes estivales (Satorre 2005; Codesido et al. 2011). Por lo tanto, es fundamental entender cómo la lechuza vizcachera responde a estos cambios inducidos por el ser humano. El objetivo del estudio es conocer el uso y la selección del hábitat de nidificación de la lechuza vizcachera en agroecosistemas de sur de la Pampa Ondulada en relación al cultivo de la soja, en tres escalas espaciales: 1) el hábitat puntual donde se localiza el nido (escala de nido), del cual depende la presencia del nido de la lechuza vizcachera; 2) el sitio de nidificación, definido por un círculo de $50 \mathrm{~m}$ de radio con centro en el nido, donde la lechuza vizcachera descansa y permanece la mayor parte de su tiempo, y 3) el área de acción, constituida por un círculo de $600 \mathrm{~m}$ de radio con centro en el nido, dentro del cual la lechuza vizcachera realiza todas sus actividades (Haug and Oliphant 1990).

Dado que el incremento de la intensidad del disturbio antrópico típicamente afecta de forma negativa a las especies nativas (Howard et al. 2001), nos preguntamos si el uso del hábitat de la lechuza vizcachera difiere antes y después del laboreo que implica el cultivo de la soja de primera en las tres escalas espaciales antes definidas. Según el momento del año en que se siembra la soja se pueden distinguir dos tipos: la soja de primera (que se siembra en octubre en la Pampa Ondulada) y la soja de segunda (que se siembra inmediatamente después de la cosecha de trigo, en diciembre o en los primeros días de enero). Los campos con soja de primera permanecen en descanso o con baja carga ganadera durante el invierno (relativamente baja intensidad de disturbio antrópico); por lo tanto, esperamos que sean hábitats aptos para nidificación al inicio de la época reproductiva de la lechuza vizcachera (antes del brote de la soja de primera). Luego, esos campos se siembran con soja, por lo que esperamos que después del brote de la soja, los bordes de campos de cultivo sean usados con mayor frecuencia por la lechuza vizcachera para anidar, ya que presentan una perturbación baja comparados con los campos de cultivo. La selección del hábitat 
de nidificación se evaluó después del brote de la soja en las tres escalas espaciales, y se compararon variables del hábitat donde estaban localizados los nidos con los puntos al azar. Esperamos que los bordes de campos de cultivo sean seleccionados para localizar el nido, ya que presentan una baja perturbación comparados con los campos de cultivo. En la escala de sitio de nidificación se espera que la lechuza vizcachera seleccione hábitats con menor perturbación, como los campos ganaderos, que proveen recursos para la alimentación y nidificación (Haug and Oliphant 1990). Además, esperamos que la lechuza vizcachera elija nidificar en sitios donde haya disponibilidad de postes de alambrado, que le permitirían mejorar su habilidad para detectar depredadores y posibles presas, ya que logran mejor visibilidad (Scobie et al. 2014). También se espera que eviten nidificar en áreas dominadas por cultivos. Por último, a escala del área de acción se espera que seleccionen áreas abiertas, ya que brindan mayor visibilidad en el forrajeo y reducen el riesgo de depredación (Villareal et al. 2005).

\section{Materiales y Métodos}

\section{Área de estudio}

El trabajo se realizó en los partidos de Exaltación de la Cruz y San Andrés de Giles (Figura 1), provincia de Buenos Aires,
Argentina. El área de estudio está localizada en la provincia fitogeográfica Pampeana, Distrito Pampeano Oriental (Cabrera 1971), en el sector sur de la Pampa Ondulada (León 1991). La comunidad nativa característica es el flechillar de Piptochaetium montevidense, Stipa neesiana, Stipa intricata, Bothriochloa laguroides, Paspalum dilatatum, Paspalum quadrifarium y Cortaderia selloana (León 1991; Soriano et al. 1991). Los pastizales naturales son poco frecuentes y corresponden sólo a superficies no cultivadas recientemente, con comunidades en equilibrio con el pastoreo (Burkart et al. 2005). Dado que esta región posee suelos profundos y ricos en materia orgánica, el reemplazo de los pastizales naturales, primero por la ganadería y luego por la agricultura, fue casi completo, lo que empobreció la materia orgánica de los suelos (Imbellone et al. 2010). En algunos campos se realizan dos cultivos anuales (generalmente trigo en invierno y soja de segunda en verano), pero es común que entre cultivos estivales sucesivos (i.e., soja, maíz, girasol) el terreno permanezca en reposo o que el ganado se alimente de las pasturas que crecen de manera espontánea (i.e., no son implantadas). En este estudio se considera a las pasturas como un tipo de hábitat que sufre menos perturbación que los campos cultivados. Los bordes de los campos (debajo y a ambos lados de los alambrados) constituyen ambientes longitudinales donde crece vegetación espontánea. En el sur de la Pampa Ondulada, la mayoría de los bordes

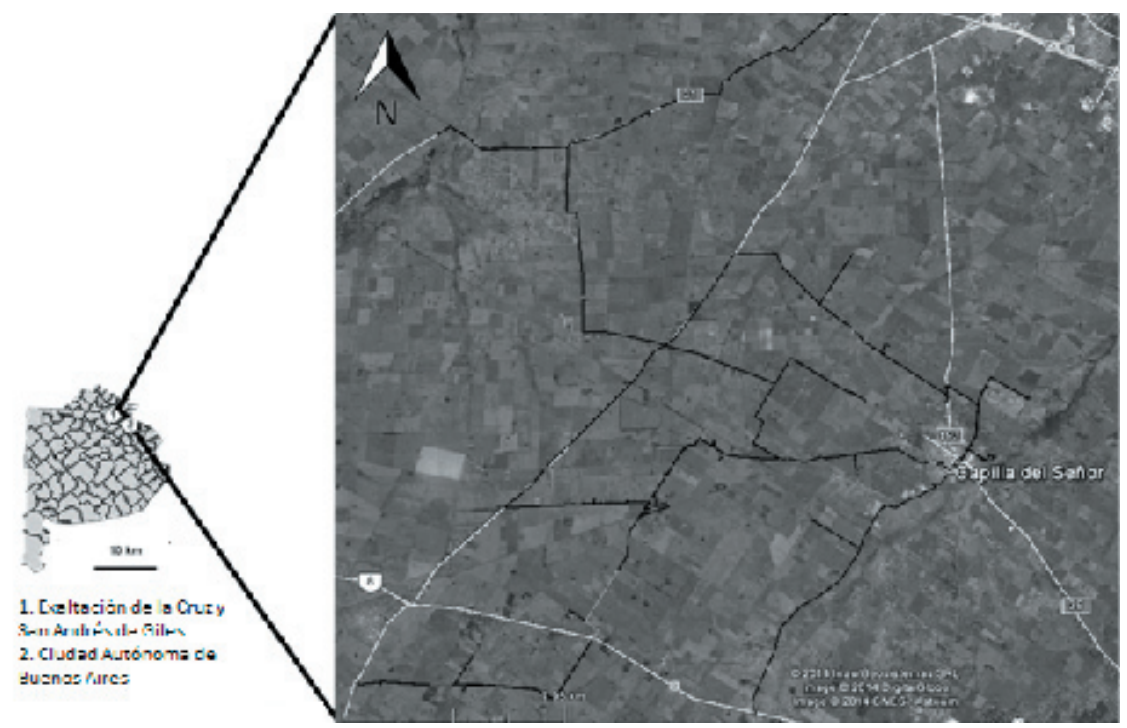

Figura 1. Localización del área de estudio en el partido de Exaltación de la Cruz y San Andrés de Giles (norte de la provincia de Buenos Aires). Se encuentra ampliada el área de estudio junto con los caminos recorridos (líneas negras) en la búsqueda de los nidos de lechuza vizcachera.

Figure 1. Location of the study area in Partido de Exaltación de la Cruz and San Andrés de Giles (north of the province of Buenos Aires). Roads used for searching nest site of Burrowing Owl are in black. 
están exentos de manejos como la aplicación de herbicidas o las quemas prescriptas. La región es casi plana, con una pendiente ligera. El clima es templado-húmedo, con veranos cálidos e inviernos fríos (Soriano et al. 1991). La temperatura media anual del área de estudio es $16{ }^{\circ} \mathrm{C}$ y la precipitación media anual es $1200 \mathrm{~mm}$ (Servicio Meteorológico Nacional).

\section{Diseño del estudio}

El trabajo de campo se llevó a cabo durante la primavera, entre octubre y diciembre de 2012. Dicho período coincide con la época de nidificación de la lechuza vizcachera (De la Peña 2010), que comienza a establecer sus territorios reproductivos antes de que empiece la siembra de la soja de primera. Utiliza el nido como sitio de refugio o de descanso durante la época no reproductiva y suele reutilizar los sitios para anidar en el período reproductivo (Machicote et al. 2004). Para localizar los nidos de las lechuzas se recorrieron $120 \mathrm{~km}$ en vehículo a baja velocidad, en caminos secundarios de tierra. Para buscar las lechuzas se usaron binoculares, ya que suelen estar la mayor parte del día posadas en las perchas. La máxima detectabilidad de la lechuza vizcachera fue $\sim 100 \mathrm{~m}$ desde el camino. Cuando una lechuza vizcachera era avistada, se buscó el nido y se lo georreferenció. El nido se consideró activo cuando se observaron una o más lechuza vizcacheras y cuando se registró el despliegue de comportamiento de defensa del mismo y/o se encontraban los regurgitados en los alrededores del nido. Se consideraron para el análisis aquellos nidos activos distanciados al menos $50 \mathrm{~m}$ entre sí. Cuando se halló más de un nido activo dentro del radio de 50 m, se eligió uno de ellos al azar.

A fin de evaluar la selección del hábitat para nidificar en las diferentes escalas espaciales, luego del brote de la soja de primera se tomaron 23 puntos al azar (puntos sin nido). La elección de los puntos al azar se realizó calculando el total de kilómetros recorridos y mediante una función generadora de números aleatorios. Se obtuvieron 23 datos de distancia correspondientes a cada punto al azar, y también se aleatorizó el lado del camino (izquierda o derecha) donde se registrarían las variables ambientales. Dado que para la búsqueda de nidos se ingresó a los campos hasta una distancia promedio de $50 \mathrm{~m}$, se eligieron al azar los 23 puntos comprendidos entre cero (camino) y $50 \mathrm{~m}$ (en el interior del campo). Se corroboró que los puntos al azar no tuvieran nidos activos, y se verificó que no hubiera ninguna lechuza vizcachera posada en los alambrados o en arbustos. La selección del hábitat de nidificación fue evaluada mediante la comparación de las variables ambientales registradas en los sitios con nido (sitios usados) versus los puntos al azar (sitios no usados).

En la escala de nido se registró el tipo de hábitat donde estaba localizado el nido o el punto al azar. Para dicha escala, el hábitat del borde se definió como el límite entre dos tipos de hábitat de un ancho de $\sim 2 \mathrm{~m}$. La cobertura de cultivos y rastrojos fueron agrupadas en la variable Agricultura, mientras que la variable Pasturas contiene campos con vegetación de crecimiento espontáneo, con y sin la presencia de ganado. Para estimar las variables ambientales se establecieron círculos de $50 \mathrm{~m}$ (escala de sitio de nidificación) y 600 m (escala de área de acción) de radio con centro en cada nido y punto al azar. En cada círculo se estimaron los valores de las variables ambientales que caracterizan el hábitat: coberturas porcentuales de cultivos (alfalfa, trigo, maíz, soja), árboles, rastrojos, áreas urbanas, caminos y pasturas (Tabla 1). La cobertura y composición de los tipos de hábitat a la escala de sitio de nidificación fue estimada por observación directa a campo, mientras que a la escala de área de acción se determinó utilizando una clasificación supervisada de imágenes satelitales SPOT 4. Se utilizó una imagen satelital del 13 de octubre del 2012 (Path 697, Row 418) para los nidos encontrados antes del brote de la soja (NABS), mientras que la otra imagen, del 8 de diciembre del 2012, para los nidos hallados después del brote de la soja (NDBS). Se empleó el programa de uso libre SOPI I 2.1 confeccionado por la CONAE para el procesamiento de las imágenes. La clasificación global de las coberturas para la imagen satelital utilizada para los NABS fue de $92 \%$. El cálculo de la clasificación global de la otra imagen satelital no se pudo realizar. Además, se registraron variables relacionadas con los requerimientos para vigilancia y defensa frente a depredadores, como son los metros totales de alambrado, distancias a los árboles más cercanos y distancia mínima al alambrado (Tabla 1).

\section{Análisis de datos}

Para evaluar la dependencia entre el tipo de hábitat y el momento de nidificación (antes o después del brote de la soja) y la selección del tipo de hábitat (comparación entre los 
Tabla 1. Descripción de las variables de hábitat medidas a las tres escalas espaciales (nido, sitio de nidificación y área de acción) para caracterizar el hábitat de la lechuza vizcachera.

Table 1. Environmental variables estimated at the scales of nest, nesting site and home range to characterize the habitat of Burrowing Owl.

\begin{tabular}{|c|c|c|}
\hline Escala & Variable & Descripción \\
\hline Nido & Tipo de hábitat & Tipo de hábitat de localización del nido o punto al azar \\
\hline \multirow{6}{*}{$\begin{array}{l}\text { Sitio de } \\
\text { nidificación }{ }^{1}\end{array}$} & Soja $(\%)$ & Porcentaje de superficie cubierta por soja de primera \\
\hline & Pasturas (\%) & $\begin{array}{l}\text { Porcentaje de las áreas con vegetación de crecimiento espontáneo } \\
\text { de hasta } 30 \mathrm{~cm} \text { de altura, con y sin ganado }\end{array}$ \\
\hline & Camino (\%) & Porcentaje de caminos secundarios de tierra y pavimentados \\
\hline & Metros totales de alambrado $(\mathrm{m})$ & Sumatoria de los metros de alambrado \\
\hline & Árboles (\%) & Porcentaje de las arboledas \\
\hline & Rastrojo (\%) & Porcentaje de áreas con residuos de la cosecha \\
\hline \multirow[t]{5}{*}{$\begin{array}{l}\text { Área de } \\
\text { acción }^{2}\end{array}$} & $\begin{array}{l}\text { Soja }(\%) \\
\text { Pasturas }(\%) \\
\text { Camino }(\%) \\
\text { Distancia mínima al alambrado } \\
(\mathrm{m}) \\
\text { Distancia a árboles más cercanos } \\
(\mathrm{m})\end{array}$ & $\begin{array}{l}- \\
- \\
\text { Distancia desde el nido o punto al azar al alambrado más } \\
\text { cercano } \\
\text { Distancia desde el nido o punto al azar al grupo de árboles más } \\
\text { cercano }\end{array}$ \\
\hline & Árboles (\%) & - \\
\hline & Cultivos (\%) & Porcentaje de los cultivos presentes a excepción de la soja \\
\hline & Urbano (\%) & Porcentaje de las áreas residenciales \\
\hline & Rastrojo (\%) & - \\
\hline
\end{tabular}

${ }^{1}$ Se refiere a un área de $50 \mathrm{~m}$ de radio con centro en el nido.

${ }^{2}$ Se refiere a un área de $600 \mathrm{~m}$ de radio con centro en el nido.

NDBS y puntos al azar) a la escala de nido se realizó la prueba exacta de Fisher, utilizada frecuentemente para tablas de contingencia de $2 \times 2$ (aunque se hace extensivo para tablas de $3 \times 2$ ) y frecuencias observadas menores a cinco. Para analizar las diferencias en las variables ambientales entre los nidos hallados antes y después del brote de la soja (NABS vs NDBS) a escala de sitio denidificación y deárea deacción se utilizó un análisis discriminante (Zuur et al. 2007). Se evaluó la multicolinealidad entre las variables explicativas mediante el cálculo de los coeficientes de Pearson; se eligió una sola variable explicativa en los casos en que el coeficiente de correlación fue mayor a 0.7. Los supuestos de homocedasticidad y normalidad multivariada de las variables ambientales fueron analizados mediante pruebas de Box y Shapiro Wilks. Para evaluar la selección del hábitat de nidificación a la escala de sitio y área de acción se utilizaron modelos lineales generalizados (Zuur et al. 2009). La variable respuesta fue la presencia-ausencia de nido, que sigue una distribución binomial ( 1 =presencia de nido; $0=$ punto al azar). Las variables explicativas fueron las definidas según la escala correspondiente en la Tabla 1, y previamente se evaluó la multicolinealidad entre las mismas, tal como se realizó para el análisis discriminante. Luego se realizó una selección de modelos a partir de un modelo con las variables explicativas finales, de tipo "backward", para la selección del modelo más parsimonioso. Para todos los análisis se utilizó el programa $R$, versión 3.3.1 ( $R$ Development Core Team 2015). Para el análisis de la selección a escala de sitio de nidificación se utilizó el paquete Logistf (Heinze et al. 2016), que realiza modelos logísticos y evita el problema de separación entre 0 y 1 de la variable respuesta mediante el método de reducción de sesgos de Firth.

\section{Resultados}

Se registraron un total de 36 nidos activos, 16 antes del brote de la soja y 20 después del brote de soja.

\section{Comparación de los sitios de nidificación antes y después del brote de la soja}

La frecuencia relativa de nidos localizados en los distintos tipos de hábitats antes del brote de la soja (escala de nido) difirió significativamente de la frecuencia de nidos encontrados después del brote de la soja $(P=0.008)$. Los NABS se encontraron principalmente en Pasturas (63\%), mientras que los NDBS se localizaron en su mayoría en los Bordes (55\%) (Figura 2A). Cabe señalar que la categoría Agricultura de los NABS 

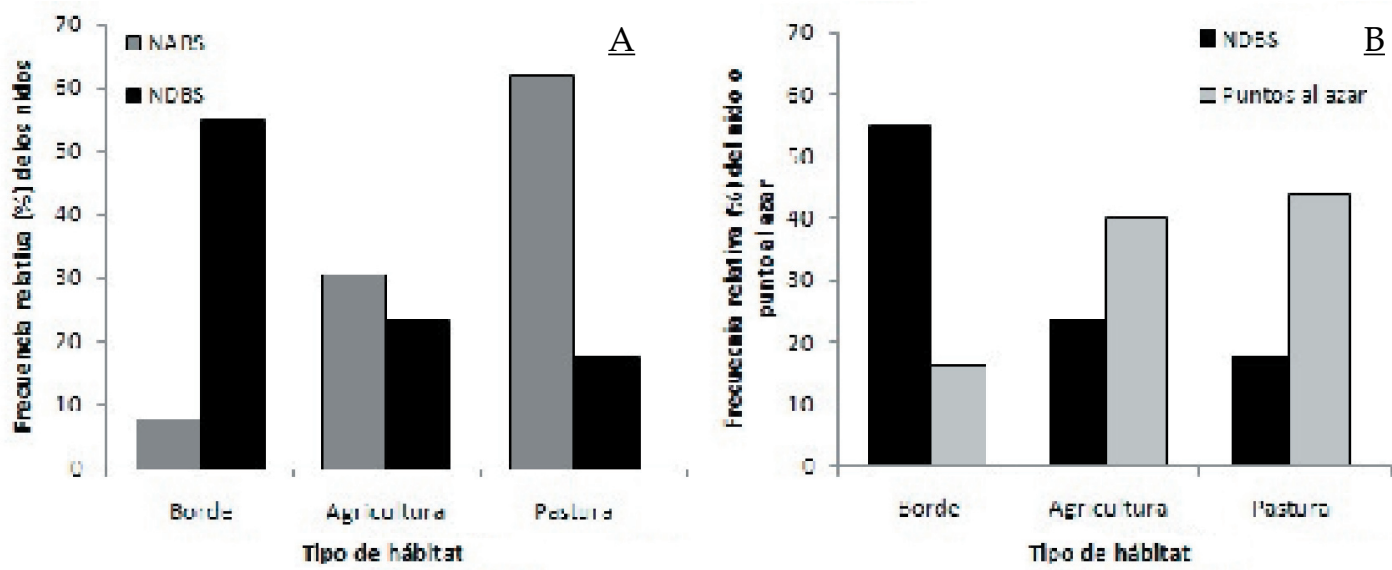

Figura 2. Frecuencia relativa (\%) de los nidos de lechuza vizcachera y de puntos al azar localizados en los distintos tipos de hábitat a escala de nido para: A) los nidos antes del brote de la soja (NABS) y después del brote de la soja (NDBS), y B) los puntos al azar y los NDBS.

Figure 2. Relative frequency (\%) of Burrowing Owls nests and random points located in different habitat types at scale of nest: A) before (NABS) and after (NDBS) the soybean shooting, and B) NDBS and random points.

está representada por $90 \%$ de rastrojos; en cambio, la misma categoría en NDBS se halla constituida por un $93 \%$ de soja. A la escala de sitio de nidificación no se encontraron diferencias significativas de acuerdo a la cobertura de Pasturas y Caminos y Metros Totales De Alambrado entre los NABS y los NDBS $(\lambda$ de Wilks $=0.94, P=0.67)$. A la escala de área de acción, los NABS y los NDBS difirieron de acuerdo a las variables ambientales consideradas en el análisis discriminante $(\lambda$ de Wilks $=0.22, P<0.001)$. Los NDBS tuvieron en promedio $25 \%$ menos cobertura de Rastrojo (ANOVA univariado, $P=0.001$ ), $44 \%$ más cobertura de Soja $(P<0.001)$ y $15 \%$ menos de cobertura de Otros cultivos $(P<0.001)$ respecto a los NABS.

\section{Selección del hábitat de nidificación después del brote de la soja}

Se encontraron diferencias significativas entre las frecuencias de los tipos de hábitat de NDBS respecto a los puntos al azar $(P=0.019)$ (Figura 2B). Luego del brote de la soja, la lechuza vizcachera nidificó en los bordes con mayor frecuencia respecto a los puntos al azar. A escala de sitio de nidificación, el modelo más parsimonioso incluyó la Cobertura de árboles y Cobertura de caminos, que estuvieron relacionadas negativamente con la presencia de la lechuza vizcachera (Tabla 2). A la escala de área de acción, el modelo final incluyó la Distancia a árboles más cercanos y la Distancia mínima al alambrado (Tabla 2). Sólo la variable Distancia a los árboles más cercanos fue significativa en el modelo, y la presencia de la lechuza vizcachera fue más probable en sitios alejados de las arboledas. Los nidos de la lechuza vizcachera estuvieron en promedio $150 \mathrm{~m}$ más alejados de las arboledas que los puntos al azar.

\section{Discusión}

\section{Uso del hábitat de nidificación}

La lechuza vizcachera cambió su uso de hábitat de nidificación en coincidencia con la fenología del cultivo dominante en la región.

Tabla 2. Variables de hábitat del modelo final con su estimador ( $\beta$ ), error estándar (SE) y el P-valor $(P)$ para la presencia / ausencia de la lechuza vizcachera en la Pampa Ondulada a la escala de sitio de nidificación y de área de acción. *indican las variables significativas de los modelos.

Table 2. Environmental variables of the final model with the estimator $(\beta)$, standard error (SE) and P-value $(P)$ for the presence/absence of the Burrowing Owl in the Rolling Pampa at the nesting site and home range scale. *indicates significant variables of models.

\begin{tabular}{llccc}
\hline Escala & Variables de hábitat & $\beta$ & Error estándar (SE) & $P$ \\
\hline \multirow{2}{*}{ Sitio de nidificación } & Camino (\%) & -0.289 & 0.124 & $<0.001^{*}$ \\
& Árboles (\%) & -0.169 & 0.103 & $0.002^{*}$ \\
\multirow{2}{*}{ Acción de acción } & Distancia mínima al alambrado (m) & -0.024 & 0.015 & 0.100 \\
& Distancia a árboles más cercanos (m) & 0.011 & 0.004 & $0.009^{*}$ \\
\hline
\end{tabular}


Nidificó en mayor frecuencia en el interior de las pasturas antes del brote de la soja de primera, mientras que luego del brote de la soja utilizó bordes de cultivos de soja. En la misma zona, Bellocq (1993) halló resultados similares antes del brote de los cultivos de verano, en donde la mayoría de los nidos fueron construidos en parcelas sin labranzas recientes, dedicadas al pastoreo de ganado o en bordes de campos y caminos. Es probable que las lechuzas que nidifiquen en esos campos relocalicen sus nidos en los bordes una vez que ingresan las máquinas para la siembra. Los campos sin labranzas recientes serían colonizados por la lechuza vizcachera para la construcción de sus nidos y, posteriormente, la puesta coincide con la siembra de los cultivos estivales, lo cual destruye los nidos (Bellocq 1993). Además, en la región ampliamente cultivada por cereales de Saskatchewan, en las praderas de Canadá, las lechuzas también evitaron las tierras de cultivo y se alimentaron en el núcleo de las pasturas (Sissons et al. 2001; Poulin et al. 2005). Los resultados a escala de nido coinciden con nuestras predicciones, ya que se esperaba un cambio en la frecuencia del hábitat de localización del nido después del disturbio ocasionado por la soja hacia ambientes con menor perturbación humana como los bordes de cultivos de soja. Nuestro estudio no evaluó si la puesta había comenzado antes del brote de la soja, en cuyo caso el costo incluye la búsqueda de un nuevo sitio para anidar $y$, eventualmente, de una segunda postura. En caso contrario, el costo de relocalizar el nido se limita a la búsqueda de un nuevo sitio para anidar.

A escala de sitio de nidificación, las lechuzas utilizaron el hábitat para la nidificación de manera similar antes y después del brote de la soja a pesar del disturbio provocado por la siembra. Este resultado es comparable al reportado por Da Silva et al. (2015) en sus estudios de uso de hábitat de aves en pastizales de Rio Grande do Sul y del norte de Uruguay, donde la lechuza vizcachera utiliza indistintamente los pastizales seminaturales y los cultivos de soja. En la escala de área de acción, los cambios observados en el uso del hábitat antes y después del brote de la soja concuerdan con el ciclo de la soja en la Pampa Ondulada: disminución del rastrojo debido a la preparación de la tierra para el cultivo de soja de segunda, aumento de soja de primera y disminución de los otros cultivos (tal vez debido a su cosecha). A pesar del disturbio generado por la soja, los resultados sugieren que la lechuza vizcachera pudo adaptarse a dichos cambios. En un estudio sobre su alimentación en hábitats naturales y modificados por el ser humano en la Pampa, Cavalli et al. (2014) encontraron que la lechuza vizcachera pudo colonizar y adaptarse fácilmente a hábitats recién modificados por el ser humano, demostrando un comportamiento plástico.

\section{Selección del hábitat de nidificación}

La lechuza vizcachera seleccionó los bordes de los cultivos como lugar para localizar el nido luego del brote de la soja de primera. Estos bordes se caracterizan por el crecimiento espontáneo de plantas herbáceas $\mathrm{y}$, además, tienen alambrados para delimitar el parcelamiento. Este resultado coincide con el encontrado por Baladrón et al. (2016) en la Pampa Inundable en la escala de paisaje (círculo de radio de $1200 \mathrm{~m}$ ), donde los bordes proporcionan mejores oportunidades de caza para la lechuza vizcachera debido al aumento en la disponibilidad de presas, asociado a la presencia de vegetación espontánea.

A la escala de sitio de nidificación, nuestro modelo final incluyó variables relacionadas con el disturbio antrópico generado en los agroecosistemas (e.g., caminos para la circulación de vehículos y la plantación de árboles en cercanías de las casas). Por un lado, los caminos pueden alterar la presencia de la lechuza vizcachera debido al disturbio ocasionado por los vehículos circulantes. Por otra parte, la lejanía de grupos de árboles permite a la lechuza vizcachera una mejor visibilidad del área que rodea a los nidos (Schmutz 1997; Ronan 2002; Thiele et al. 2013), y es un resultado esperado, ya que es una especie típica de pastizales. De la misma manera, los avistamientos de la lechuza vizcachera en campos agrícolas del sureste de Phoenix (Estados Unidos) fueron más frecuentes en áreas con árboles dispersos que en áreas boscosas (Beebe et al. 2014). Es llamativa la ausencia de una relación significativamente positiva de los Metros Totales de Alambrado en la selección de nidos, lo cual podría deberse a que no es un recurso limitante en los agroecosistemas. También cabe destacar que la selección del sitio de nidificación no estuvo negativamente influenciada por el cultivo de soja. Algunos autores proponen que la cercanía a los campos de cultivo disminuye el riesgo de depredación de los nidos (Orth and Kennedy 2001). Sin 
embargo, la presencia de la lechuza vizcachera en la Pampa Inundable estuvo asociada de manera negativa a los cultivos a la escala de territorio (la equivalente a la escala de área de acción) (Baladrón et al. 2016). En ese caso, el área de estudio incluyó no sólo cultivos, sino también pastizales, campos de pastoreo, dunas vegetadas o sin vegetación y áreas urbanas. En contraste, nuestra área de estudio tiene una matriz de paisaje dominada por un uso de la tierra con una fuerte intensidad de disturbio ocasionada por el cultivo de soja. Esto implicaría una mejor adaptación de la lechuza a la estructura de paisaje de la Pampa Ondulada y una selección diferencial de hábitat dependiente de la región.

En la escala de área de acción, la lechuza vizcachera prefirió nidificar cerca de los alambrados y evitó hacerlo cerca de grupos de árboles. Este resultado coincide con nuestra predicción. Las lechuzas podrían estar seleccionando nidificar cerca de postes de alambrado debido a que son buenos sitios de observación para detectar presas y vigilar a posibles depredadores y/o podría ser el resultado del alejamiento al disturbio generado por la siembra. También, podría ocurrir que los sitios cerca de los alambrados fueran lo único disponible, no disturbado ni cultivado, que la lechuza vizcachera tiene para nidificar. Es necesario destacar que la probabilidad de detectar las lechuzas posadas en los postes de alambrados es mayor que la probabilidad de encontrarlas posadas en el suelo, lejos de los postes. Por lo tanto, los resultados encontrados en relación a esta variable deben ser tomados con precaución.

Muchos estudios enfatizan la importancia del análisis a múltiples escalas (Wiens 1981; Stevens et al. 2011; Thiele et al. 2013). Este trabajo demostró que la lechuza vizcachera selecciona variables relacionadas al hábitat de nidificación que se expresan a distintas escalas espaciales en los agroecosistemas pampeanos, y se comporta como una especie generalista de áreas abiertas, capaz de adaptarse al disturbio que ocasiona la agricultura de la región. Por el contrario, especies de aves especialistas típicas de pastizal se ven afectadas por la expansión de la actividad agrícola, ya que disminuye su abundancia y su área de ocupación (Codesido et al. 2012). La disponibilidad de postes de alambrado en agroecosistemas es mayor en comparación a otros ambientes (Martínez et al. 2017), lo cual favorecería el establecimiento de la lechuza vizcachera respecto a otras especies de aves. La lechuza vizcachera demostró tener plasticidad para establecer sus nidos en una variedad de hábitats modificados por el ser humano en los agroecosistemas pampeanos. Sin embargo, nuestros resultados sugieren que es conveniente mantener la vegetación espontánea en los bordes de los campos de cultivo para proteger los hábitats seleccionados para nidificar. Numerosas especies de aves usan el borde y también otros ambientes no cultivados (e.g., bajos, banquinas y terrazas) (Leveau and Leveau 2004; Solari and Zaccagnini 2009; Di Giacomo and López de Casenave 2010). Queda pendiente para futuros estudios evaluar el éxito reproductivo de la lechuza vizcachera en los distintos hábitats de la región Pampeana para brindar más elementos que contribuyan a la conservación de esta especie y de otras aves típicas de pastizales.

Agradecimientos. A la CONAE por proporcionar las imágenes satelitales que se utilizaron en este trabajo, a Eufracio Pairo y Carolina Pinto por la colaboración en el trabajo de campo, y a los productores y encargados de los campos que nos permitieron el acceso a sus propiedades. Se agradece a los revisores anónimos y al editor por proporcionar comentarios útiles que ayudaron a mejorar la calidad del manuscrito. Este trabajo fue financiado por la Agencia Nacional de Promoción Científica y Tecnológica de Argentina, el Consejo Nacional de Investigaciones Científicas y Técnicas de Argentina y la Universidad de Buenos Aires.

\section{REFERENCIAS}

Andrade, A., M. J. Nabte, and M. E. Kun. 2010. Diet of the burrowing owl (Athene cunicularia) and its seasonal variation in Patagonian steppes: implications for biodiversity assessments in the Somuncurá Plateau Protected Area, Argentina. Studies on Neotropical Fauna and Environment 45:101-110. DOI: 10.1080/01650521.2010.502010.

Baladrón, A. V., J. P. Isacch, M. Cavalli, and M. S. Bó. 2016. Habitat selection by Burrowing Owls (Athene cunicularia) in the Pampas of Argentina: a multiple-scale assessment. Acta Ornithologica 51(2):137-150. DOI: 10.3161/ 00016454AO2016.51.2.001.

Beebe, S. R., A. B. Switalski, H. L. Bateman, and K. D. Hristovski. 2014. Burrowing owl (Athene cunicularia) habitat associations in agriculture fields and along canal trails in Phoenix, Arizona. Journal of the Arizona-Nevada Academy of Science 45(2):52-58. DOI: 10.2181/036.045.0206.

Bellocq, M. I. 1987. Selección de hábitat de caza y depredación diferencial de Athene cunicularia sobre roedores en 
ecosistemas agrarios. Revista Chilena de Historia Natural 60:81-86.

Bellocq, M. I. 1988. Dieta de Athene cunicularia (Aves, Strigidae) y sus variaciones estacionales en ecosistemas agrarios de la Pampa, Argentina. Physis, Sección C 46:17-22.

Bellocq, M. I. 1993. Reproducción, crecimiento y mortalidad de la lechucita vizcachera (Speotyto cunicularia) en agroecosistemas pampeanos. Hornero 13(04):272-276.

Block, W. M., and L. A. Brennan. 1993. The habitat concept in ornithology: Theory and applications. Current Ornithology 11:35-91.

Boscolo, D., and J. P. Metzger. 2009. Is bird incidence in Atlantic forest fragments influenced by landscape patterns at multiple scales? Landscape Ecology 24(7):907-918. DOI: 10.1007/s10980-009-9370-8.

Burkart, S. E., M. F. Garbulsky, C. M. Ghersa, J. P. Guerschman, R. J. León, M. Oesterheld and S. B. Perelman. 2005. Las comunidades potenciales del pastizal pampeano bonaerense. La heterogeneidad de la vegetación de los agroecosistemas: Un homenaje a Rolando León. Editorial Facultad de Agronomía. Buenos Aires, Argentina.

Cabrera, A. L. 1971. Fitogeografía de la República Argentina. Boletín de la Sociedad Argentina de Botánica 14:1-42.

Canevari, M., P. Canevari, G. Carrizo, G. Harris, J. Rodríguez Mata, and R. Straneck. 1991. Nueva guía de las aves argentinas. Tomo 2. Fundación Acindar, Bs. As., Argentina.

Carevic, F. S., E. R. Carmona, and A. Muñoz-Pedreros. 2013. Seasonal diet of the Burrowing owl Athene cunicularia Molina, 1782 (Strigidae) in a hyperarid ecosystem of the Atacama Desert in northern Chile. Journal of Arid Environments 97: 237-241. DOI: 10.1016/j.jaridenv.2013.07.008

Cavalli, M., A. V. Baladrón, J. P. Isacch, G. Martínez, and M. S. Bó. 2014. Prey selection and food habits of breeding Burrowing owls (Athene cunicularia) in natural and modified habitats of Argentine Pampas. Emu 114(2):184-188. DOI: $10.1071 /$ MU13040.

Chipman, E. D., N. E. Mcintyre, R. E. Strauss, M. C. Wallace, J. D. Ray, and C. W. Boal. 2008. Effects of human land use on western Burrowing owl foraging and activity budgets. Journal of Raptor Research 42(2):87-98. DOI: 10.3356/ JRR-07-20.1.

Codesido, M., C. González-Fischer, and D. Bilenca. 2011. Distributional changes of landbird species in agroecosystems of Central Argentina. The Condor 113(2):266-273. DOI: 10.1525/cond.2011.090190.

Codesido, M., C. González-Fischer, and D. Bilenca. 2012. Agricultural land-use, avian nesting and rarity in the Pampas of central Argentina. Emu 112:46-54. DOI: 10.1071/MU11049.

Conway, C. J., V. García, M. D. Smith, L. A. Ellis, and J. Whitney. 2006. Comparative demography of Burrowing owls within agricultural and urban landscapes in Southeastern Washington. Journal of Field Ornithology 77:280-290. DOI: 10.HH/j.1557-9263.2006.00054.x.

Crowe D. E., and K. M. Longshore. 2013. Nest site characteristics and nesting success of the Western Burrowing Owl in the eastern Mojave Desert. Journal of Arid Environments 94:113-120. DOI 10.1016/j.jaridenv.2013.03.004.

Cueto, V. R. 2006. Escalas en ecología: su importancia para el estudio de la selección de hábitat en aves. Hornero 21(01):1-13.

Da Silva, T. W., G. Dotta, D. T. Gressler, and C. S. Fontana. 2015. Habitat use by grassland birds in natural areas and soybean fields in southern Brazil and Uruguay. Wilson Journal of Ornithology 127(2):212-221. DOI: 10.1676/wils127-02-212-221.1.

De la Peña, M. R. 2010. Nidos de aves argentinas. CD-ROM. Universidad Nacional del Litoral, Santa Fe, Argentina.

De Tommaso, D. C., R. G. Callicó Fortunato, P. Teta, and J. A. Pereira. 2009. Dieta de la lechucita vizcachera (Athene cunicularia) en dos áreas con diferente uso de la tierra en el centro-sur de la Provincia de la Pampa, Argentina. Hornero 24(2):87-93.

Di Giacomo, A. S. D., and J. Lopez de Casenave. 2010. Use and importance of crop and field-margin habitats for birds in a Neotropical agricultural ecosystem. The Condor 112(2):283-293.

Haug, E. A., and L. W. Oliphant. 1990. Movements, activity patterns and habitat use of Burrowing owls in Saskatchewan. Journal of Wildlife Management 54:27-35. DOI: 10.1525/cond.2010.090039.

Haug, E. A., B. A. Millsap, and M. S. Martell. 1993. Burrowing Owl (Speotyto cunicularia). In A. Poole and F. Gill (eds.). The birds of North America. The Academy of Natural Sciences, Philadelphia, Pennsylvania and the American Ornithologists' Union, Washington DC, USA.

Heinze, G., M. Ploner, D. Dunkler, and H. Southworth. 2016. Logistf: Firth's bias reduced logistic regression. R package version 1 .

Holroyd, G. R., E. Rodríguez, and S. Sheffield. 2001. Conservation of the Burrowing Owl in Western North America: issues, challenges, and recommendations. Journal Raptor Research 35:399-407.

Howard M. N., S. K. Skagen, and P. L. Kennedy. 2001. Does habitat fragmentation influence nest predation in the shortgrass prairie? The Condor 103:530-536. DOI: 10.1650/0010-5422(2001)103[0530:DHFINP]2.0.CO;2.

Hudson, W. H. 1920. Birds of La Plata (Vol. 2). J. M. Dent \& Sons Limited, London, England.

Imbellone, P. A., J. E. Giménez, and J. L. Panigatti. 2010. Suelos de la Región Pampeana: procesos de formación. INTA, Instituto de Geomorfología y Suelos, Facultad de Ciencias Naturales y Museo, Universidad Nacional de La Plata, Buenos Aires, Argentina.

Johnson, D. H. 1980. The comparison of usage and availability measurements for evaluating resource preference. Ecology 61:65-71. DOI: 10.2307/1937156.

Jones, J. 2001. Habitat selection studies in avian ecology: a critical review. The Auk 118(2):557-562. 
Jones, Z. F., and C. E. Bock. 2002. Conservation of grassland birds in an urbanizing landscape: a historical perspective. The Condor 104(3):643-651. DOI: 10.1650/0010-5422(2002)104[0643:COGBIA]2.0.CO;2.

Lantz, S. J., C. J. Conway, and S. H. Anderson. 2007. Multiscale habitat selection by burrowing owls in black-tailed prairie dog colonies. Journal of Wildlife Management 71(8):2664-2672. DOI: 10.2193/2006-221.

León, R. J. C. 1991. Geographic limits of the region, geomorphology and geology, regional subdivisions, floristic aspects, description of the vegetation. Pp. 369-387 in Natural Grasslands, Introduction and Western Hemisphere. Elsevier, Amsterdam, Netherlands.

Leveau, L. M., and C. M. Leveau. 2004. Riqueza y abundancia de aves en agroecosistemas pampeanos durante el período post-reproductivo. Ornitología Neotropical 15:371-380.

Machicote, M., L. C. Branch, and D. Villarreal. 2004. Burrowing owls and burrowing mammals: are ecosystem engineers interchangeable as facilitators? Oikos 106:527-535. DOI: 10.1111/j.0030-1299.2004.13139.x.

Manly, B. F. J., M. C. Donald, and D. L. Thomas. 1993. Resource selection by animals. Statistical design aim analysis for field studies. Chapman and Hall, London, England.

Martinelli, A. G. 2010. Observaciones sobre selección y reutilización de sitios de nidificación de la lechucita de las vizcacheras Athene cunicularia (Strigiformes: Strigidae) en el Parque Municipal do Sabiá, Uberlândia, Estado de Minas Gerais, Brasil. Nótulas Faunísticas 50:1-6.

Martínez, G., A. V. Baladrón, M. Cavalli, M. S. Bó, and J. P. Isacch. 2017. Microscale nest-site selection by the Burrowing Owl (Athene cunicularia) in the pampas of Argentina. The Wilson Journal of Ornithology 129(1):62-70. DOI: 10.1676/ 1559-4491-129.1.62.

Orth, P. B., and P. L. Kennedy. 2001. Do land-use patterns influence nest-site selection by Burrowing owls (Athene cunicularia hypugaea) in Northeastern Colorado? Canadian Journal of Zoology 79:1038-1045. DOI: 10.1139/z01-071.

Palma, R. E., J. J. Polol, R. D. Owen, and J. N. Mills. 2012. Ecology of rodent-associated hantaviruses in the southern cone of South America: Argentina, Chile, Paraguay, and Uruguay. Journal of Wildlife Diseases 48:267-281. DOI: 10.7589/0090-3558-48.2.267.

Piorecky, M. D., and D. R. Prescott. 2006. Multiple spatial scale logistic and autologistic habitat selection models for northern pygmy owls, along the eastern slopes of Alberta's Rocky Mountains. Biological Conservation 129(3):360371. DOI: 10.1016/j.biocon.2005.11.003.

Poulin, R. G., L. D. Todd, K. M. Dohms, R. M. Brigham and T. I. Wellicome. 2005. Factors associated with nest- and roost-burrow selection by Burrowing owls (Athene cunicularia) on the Canadian prairies. Canadian Journal of Zoology 83:1373-1380. DOI: 10.1139/Z05-134.

R Development Core Team 2016. R: A Language and Environment for Statistical Computing. R foundation for Statistical Computing, Vienna. URL: http://www.r-project.org.

Ronan, N. A. 2002. Habitat selection, reproductive success and site fidelity of burrowing owls in a grassland ecosystem. Tesis de Maestría, Oregon State University, Corvallis, USA. Pp. 68.

Satorre, E. H. 2005. Cambios tecnológicos en la agricultura argentina actual. Ciencia Hoy 87:24-31.

Schmutz, J. K. 1997. Selected microhabitat variables near nests of Burrowing Owls compared to unoccupied sites in Alberta. Journal of Raptor Research Report 9:80-83.

Scobie, C., E. Bayne, and T. Wellicome. 2014. Influence of anthropogenic features and traffic disturbance on Burrowing Owl diurnal roosting behavior. Endangered Species Research 24:73-83. DOI: 10.3354/esr00577.

Sissons, R. A., K. Scalise, and T. I. Wellicome. 2001. Nocturnal foraging and habitat use by male burrowing owls in a heavily cultivated Region of Southern Saskatchewan. Journal of Raptor Research 35:304-309.

Solari, L. M., and M. E. Zaccagnini. 2009. Efecto de bordes arbóreos y terrazas sobre la riqueza y densidad de aves en lotes de soja de Entre Ríos, Argentina. Bioscriba 2:90-100.

Soriano, A., R. J. C. León, O. E. Sala, R. S. Lavado, V. A. Deregibus, M. A. Cauhépé, and J. H. Lemcoff. 1991. Río de la Plata Grasslands. Pp. 367-407 in R. T. Coupland (ed.). Ecosystems of the World 8A. Natural Grasslands. Introduction and Western Hemisphere.

Stevens, A. F. J., E. M. Bayne, and T. I. Wellicome. 2011. Soil and climate are better than biotic land cover for predicting home-range habitat selection by endangered Burrowing owls across the Canadian Prairies. Biological Conservation 144(5):1526-1536. DOI: 10.1016/j.biocon.2010.10.032.

Thiele, J. P., K. K. Bakker, and C. D. Dieter. 2013. Multiscale nest site selection by Burrowing owls in Western South Dakota. The Wilson Journal of Ornithology 125(4):763-774. DOI: 10.1676/13-013.1.

Thiollay, J. M., and Z. Rahman. 2002. The raptor community of Central Sulawesi: habitat selection and conservation status. Biological Conservation 107(1):111-122.

Villareal, D., M. Machicote, L. C. Branco, J. J. Martínez, and A. Gopar. 2005. Habitat patch size and local distribution of Burrowing owls (Athene cunicularia) in Argentina. Ornitología Neotropical 16:529-537.

Wiens, J. A. 1981. Scale problems in avian censusing. Studies in Avian Biology 6:513-521

Zuur, A., E. N. Ieno, and G. M. Smith. 2007. Analyzing ecological data. Springer Science and Business Media, New York, USA.

Zuur, A. F., E. N. Ieno, N. J. Walker, A. A. Saveliev, and G. M. Smith. 2009. Mixed effects models and extensions in ecology with R. Springer, New York, USA. 